\title{
SOME REMARKS ABOUT MEASURABLE PARAMETRIZATIONS
}

\author{
ROMAN POL
}

\begin{abstract}
A result about measurable selections is derived from the classical Yankov-von Neumann selection theorem which yields two theorems about parametrizations of analytic and Borel sets in the plane due to Cenzer and Mauldin [CM] and Srivatsa $[\mathbf{S}]$, respectively.
\end{abstract}

1. Introduction. Our terminology follows $[\mathbf{K}]$. Given a separable metrizable space $X$, let $\mathscr{B}(X)$ be the $\sigma$-algebra of Borel sets in $X$, let $\mathscr{B} \mathscr{A}(X)$ be the $\sigma$-algebra in $X$ generated by the analytic subsets of $X$, and let $\mathscr{B} \mathscr{A}(X) \otimes \mathscr{B}(X)$ be the $\sigma$-algebra in the square $X \times X$ generated by the rectangles $V \times W$, where $V \in \mathscr{B} \mathscr{A}(X)$ and $W \in \mathscr{B}(X)$. We denote by $2^{\omega}$ the Cantor set and $I=[0,1]$ is the unit interval. If $S \subset X \times Y$, then $S(x)=\{y:(x, y) \in S\}$ is the vertical section of $S$ at $x$.

The following two theorems were proved by D. Cenzer and R. D. Mauldin [CM] and V. V. Srivatsa $[\mathbf{S}]$, respectively.

1.1. Theorem (CEnzer And Mauldin). Let $A \subset 2^{\omega} \times 2^{\omega}$ be an analytic set whose each vertical section $A(x)$ is uncountable. Then there exists a one-to-one map $k$ of $2^{\omega} \times 2^{\omega}$ into $A$ such that:

(i) if $E \in \mathscr{B} \mathscr{A}\left(2^{\omega}\right) \otimes \mathscr{B}\left(2^{\omega}\right)$, then both $k(E)$ and $k^{-1}(E)$ are in $\mathscr{B} \mathscr{A}\left(2^{\omega}\right) \otimes \mathscr{B}\left(2^{\omega}\right)$, and

(ii) for each $x, k(x, \cdot)$ is a homeomorphism of $2^{\omega}$ into $\{x\} \times A(x)$.

1.2. TheORem (SRIVATSA). Let $B \subset I \times I$ be a Borel set whose each vertical section $B(x)$ is uncountable. Then there exists a one-to-one map $k$ of $I \times I$ onto $B$ such that:

(i) if $E \in \mathscr{B} \mathscr{A}\left(2^{\omega}\right) \otimes \mathscr{B}\left(2^{\omega}\right)$, then both $k(E)$ and $k^{-1}(E)$ are in $\mathscr{B} \mathscr{A}\left(2^{\omega}\right) \times \mathscr{B}\left(2^{\omega}\right)$, and

(ii) for each $x, k(x, \cdot)$ is a Borel isomorphism of I onto $\{x\} \times B(x)$.

The aim of this note is to derive from the classical Yankov-von Neumann selection theorem [D, Theorem 7] a proposition (Theorem 2.1) which easily yields both of these theorems. In fact, the way we prove the theorems follows closely the line of reasoning of Cenzer and Mauldin. However, while Cenzer and Mauldin used some ideas of the proof of the Yankov-von Neumann theorem in their proof of Theorem 1.1 (which made the proof rather involved and caused some obstacles to

Received by the editors December 20, 1983.

1980 Mathematics Subject Classification. Primary 28A20; Secondary 04A15.

01985 American Mathematical Society $0002-9939 / 85 \$ 1.00+\$ .25$ per page 
obtain the full strength of Theorem 1.2), we apply directly the Yankov-von Neumann theorem to an appropriate function space.

1.3. ReMARK. It should be pointed out that Theorem 1.2 is a particular case of some deep results of Srivatsa [S] concerning "Carathéodory parametrizations" of sets in product spaces. However, in his proofs Srivatsa used some methods of the "effective descriptive set theory".

It seems that the function space method we used in this note cannot be applied directly to obtain these stronger results, the main difficulty being that one should consider in that case function spaces on noncompact sets.

2. An application of the Yankov-von Neumann selection theorem. Let $\mathbf{H}\left(2^{\omega}\right)$ be the space of homeomorphic embeddings of the Cantor set $2^{\omega}$ into itself endowed with the compact-open topology, which makes $\mathbf{H}\left(2^{\omega}\right)$ a separable, completely metrizable space.

2.1. TheOREM. Let $A \subset 2^{\omega} \times 2^{\omega}$ be an analytic set whose each vertical section $A(x)$ is uncountable. Then there exists a $\mathscr{B} \mathscr{A}\left(2^{\omega}\right)$-measurable map $F: 2^{\omega} \rightarrow \mathbf{H}\left(2^{\omega}\right)$ such that $F(x)\left(2^{\omega}\right) \subset A(x)$ for each $x \in 2^{\omega}$.

Proof. At first, following Lemma 5 of Cenzer and Mauldin [CM], we replace, in some sense, the set $A$ by a $G_{\delta}$-set $B$, closed in a $G_{\delta}$-subset $2^{\omega} \times P$ of $2^{\omega} \times 2^{\omega}$.

Let $w$ : $2^{\omega} \rightarrow 2^{\omega} \times 2^{\omega}$ be a continuous map such that for some $G_{\delta}$-set $P \subset 2^{\omega}$, $w(P)=A$ (to get $w$, consider a continuous function $f$ which maps a subspace of $2^{\omega}$ homeomorphic to irrationals onto $A$, consider the closure of the graph $G(f)$ of $f$ in the space $2^{\omega} \times 2^{\omega} \times 2^{\omega}$ and take as $w$ the restriction to $\overline{G(f)}$ of the projection parallel to the first axis, $\overline{G(f)}$ being homeomorphic to $\left.2^{\omega}\right)$. Let

$$
w(x)=(u(x), v(x)), \quad B=\{(u(x), x): x \in P\} .
$$

The set $B$ is relatively closed in $2^{\omega} \times P$ and

$$
\text { for each } x \in 2^{\omega}, v \text { maps } B(x) \text { onto } A(x) \text {. }
$$

Let us put

$$
\Gamma=\left\{(x, h) \in 2^{\omega} \times \mathbf{H}\left(2^{\omega}\right): h\left(2^{\omega}\right) \subset B(x), \text { and } v \circ h \in \mathbf{H}\left(2^{\omega}\right)\right\},
$$

and let us check that the set

$$
\Gamma \text { is analytic and } \Gamma(x) \neq \varnothing \text { for } x \in 2^{\omega} \text {. }
$$

Let us notice that the map $v^{*}: \mathbf{H}\left(2^{\omega}\right) \rightarrow \mathbf{C}\left(2^{\omega}, 2^{\omega}\right)$, defined by the formula

$$
v^{*}(h)=v \circ h \text { is continuous, }
$$

where $\mathbf{C}\left(2^{\omega}, 2^{\omega}\right)$ is the space of continuous functions of the Cantor set into itself endowed with the compact-open topology, and hence the set

$$
\mathbf{G}=\left\{h \in \mathbf{H}\left(2^{\omega}\right): v \circ h \in \mathbf{H}\left(2^{\omega}\right)\right\}=\left(v^{*}\right)^{-1}\left(\mathbf{H}\left(2^{\omega}\right)\right),
$$

is $G_{\delta}$ in $\mathbf{H}\left(2^{\omega}\right)$. If $Q$ is a countable, dense subset of $P$, then, given $h \in \mathbf{H}\left(2^{\omega}\right)$, the inclusion $h\left(2^{\omega}\right) \subset B(x)$ means exactly that $h\left(2^{\omega}\right) \subset P$ and for each $q \in Q, h(q) \in$ $B(x), B(x)$ being closed in $P$. Thus

$$
\begin{aligned}
\Gamma=\bigcap_{q \in Q} \operatorname{proj}\left\{(x, h, y) \in 2^{\omega} \times \mathbf{H}\left(2^{\omega}\right) \times 2^{\omega}: h \in \mathbf{G},\right. \\
\left.h\left(2^{\omega}\right) \subset P,(x, y) \in B, y=h(q)\right\},
\end{aligned}
$$


where proj is the projection parallel to the third axis, and hence $\Gamma$ is an analytic set, the sets on the right-hand side being analytic.

To see that $\Gamma(x) \neq \varnothing$, choose (see (1)) a Cantor set $C \subset B(x)$ such that the restriction of $v$ to $C$ is one-to-one and take a homeomorphism $h: 2^{\omega} \rightarrow C$; then $(x, h) \in \Gamma$.

Let us apply now the Yankov-von Neumann selection theorem [D, Theorem 7] to the analytic set $\Gamma$ : there exists a $\mathscr{B} \mathscr{A}\left(2^{\omega}\right)$-measurable map $s: 2^{\omega} \rightarrow \mathbf{H}\left(2^{\omega}\right)$ such that $(x, s(x)) \in \Gamma$ for each $x \in 2^{\omega}$. Since $v^{*}$ (see (4)) is continuous, the composition $v^{*} \circ s$ is $\mathscr{B} \mathscr{A}\left(2^{\omega}\right)$-measurable and therefore the map $F(x)=v \circ s(x)$ satisfies the assertions of the theorem (cf. (1) and (2)).

The following corollary asserts a little bit more than Theorem 1.1.

2.2. Corollary. Let $A \subset 2^{\omega} \times 2^{\omega}$ and $F: 2^{\omega} \rightarrow \mathbf{H}\left(2^{\omega}\right)$ be as in Theorem 2.1. Define a map $k: 2^{\omega} \times 2^{\omega} \rightarrow A$ by the formula

$$
k(x, y)=(x, F(x)(y)) .
$$

Then, for each natural number $n$, if $E \in \mathscr{B} \mathscr{A}\left(2^{\omega}\right) \otimes \mathscr{B}\left(2^{\omega}\right)$, then both $k^{n}(E)$ and $\left(k^{n}\right)^{-1}(E)$ belong to $\mathscr{B} \mathscr{A}\left(2^{\omega}\right) \otimes \mathscr{B}\left(2^{\omega}\right), k^{n}$ being the nth iterate of $k$.

Proof. Fix a natural number $n$ and put

$$
u(x)=F^{n}(x), \quad k^{n}(x, y)=(x, u(x)(y))=v(x, y)
$$

$$
u: 2^{\omega} \rightarrow \mathbf{H}\left(2^{\omega}\right) \text { is } \mathscr{B} \mathscr{A}\left(2^{\omega}\right) \text {-measurable, }
$$

being a composition of the $\mathscr{B} \mathscr{A}\left(2^{\omega}\right)$-measurable map $x \rightarrow(F(x), \ldots, F(x))$ and the continuous superposition operation $\left(h_{1}, \ldots, h_{n}\right) \rightarrow h_{1} \circ \ldots \circ h_{n}$.

Let $V \times W$ be a rectangle with $V \in \mathscr{B} \mathscr{A}\left(2^{\omega}\right)$ and $W$ closed-and-open in $2^{\omega}$; since $\mathscr{B} \mathscr{A}\left(2^{\omega}\right) \otimes \mathscr{B}\left(2^{\omega}\right)$ is generated by such rectangles, it is enough to show that

$$
v^{-1}(V \times W) \text { and } v(V \times W) \text { are in } \mathscr{B} \mathscr{A}\left(2^{\omega}\right) \otimes \mathscr{B}\left(2^{\omega}\right) .
$$

Let $K_{1}, K_{2}, \ldots$ be the collection of all closed-and-open sets in $2^{\omega}$ and let $\mathbf{H}_{i}=\left\{h \in \mathbf{H}\left(2^{\omega}\right): h\left(K_{i}\right) \subset W\right\} ;$ since $\mathbf{H}_{i}$ are open in $\mathbf{H}\left(2^{\omega}\right), E_{i}=V \cap u^{-1}\left(\mathbf{H}_{i}\right) \in$ $\mathscr{B} \mathscr{A}\left(2^{\omega}\right)$, by (5), and hence $v^{-1}(V \times W)=\bigcup_{i} E_{i} \times K_{i} \in \mathscr{B} \mathscr{A}\left(2^{\omega}\right) \otimes \mathscr{B}\left(2^{\omega}\right)$.

The verification of the second part of (6) is also standard (cf. [K, p. 378; D, Theorem 18; S, Lemma 3.4]).

Put $E=v(V \times W)$ and let $P(G)$ be the projection of the set $E \cap\left(2^{\omega} \times G\right)$ onto the first axis for each open set $G \subset 2^{\omega}$. Let $Q$ be a countable set dense in $2^{\omega}$ and let $f_{q}(x)=u(x)(q)$; since $u(x) \in \mathbf{H}\left(2^{\omega}\right)$ and $f_{q}$ are $\mathscr{B} \mathscr{A}\left(2^{\omega}\right)$-measurable (see (5)), the set $P(B)=V \cap \bigcup\left\{f_{q}^{-1}(B): q \in Q \cap W\right\}$ is in $\mathscr{B} \mathscr{A}\left(2^{\omega}\right)$. Now, if $\mathscr{G}_{n}$ is a finite open cover of $2^{\omega}$ by sets of diameter $\leqslant 1 / n$, and if we let $E_{n}=\bigcup\left\{P(G) \times G: G \in \mathscr{G}_{n}\right\}$, we get $E=\bigcap_{n} E_{n}$, as the vertical sections $E(x)=u(x)(W)$ are closed in $2^{\omega}$, and hence $E \in \mathscr{B} \mathscr{A}\left(2^{\omega}\right) \otimes \mathscr{B}\left(2^{\omega}\right)$.

3. Proof of Theorem 1.2. To make this note complete, we shall repeat a SchröderBernstein type argument due to Cenzer and Mauldin [CM, Proof of Theorem 4] to derive Theorem 1.2 from Corollary 2.2. 
Let $B \subset 2^{\omega} \times 2^{\omega}$ be a Borel set whose vertical sections $B(x)$ are all uncountable, let $k: 2^{\omega} \times 2^{\omega} \rightarrow B$ be the map described in Corollary 2.2, let

$$
\begin{gathered}
S_{0}=B \backslash k\left(2^{\omega} \times 2^{\omega}\right), \quad T_{0}=\left(2^{\omega} \times 2^{\omega}\right) \backslash B, \\
S_{n}=k^{n}\left(S_{0}\right), \quad T_{n}=k^{n}\left(T_{0}\right), \quad D=\bigcap_{n=1}^{\infty} k^{n}\left(2^{\omega} \times 2^{\omega}\right),
\end{gathered}
$$

and let

$$
\begin{aligned}
H= & D \cup \bigcup_{n=1}^{\infty} S_{n}, \quad G=\bigcup_{n=1}^{\infty} T_{n}, \\
& g(z)\left\{\begin{array}{l}
z \text { if } z \in H, \\
k(z) \quad \text { if } z \in G .
\end{array}\right.
\end{aligned}
$$

Since $S_{i}, T_{i}$ and $D$ are in $\mathscr{B} \mathscr{A}\left(2^{\omega}\right) \otimes \mathscr{B}\left(2^{\omega}\right)$ (in particular, their vertical sections are Borel), the map $g$ is a $\mathscr{B} \mathscr{A}\left(2^{\omega}\right) \otimes \mathscr{B}\left(2^{\omega}\right)$-isomorphism of $2^{\omega} \times 2^{\omega}$ onto $B$ such that each $g(x, \cdot)$ is a Borel isomorphism of $2^{\omega}$ onto $\{x\} \times B(x)$. Of course, $2^{\omega}$ can be replaced here by $I$, or by any other uncountable, separable complete metric space-all of them being Borel isomorphic.

\section{Comments.}

4.1. R. D. Mauldin [M, Theorem 1] proved the following

Theorem (MAUldin). Let $A \subset 2^{\omega} \times 2^{\omega}$ be an analytic set such that the set $\left\{x \in 2^{\omega}: A(x)\right.$ is uncountable $\}$ is uncountable. Then there exists a (antol ' $C \subset \operatorname{proj} A$ and a homeomorphism $g$ of $C \times 2^{\omega}$ into A such that $g(x, \cdot): 2^{\omega} \rightarrow\{x\} \times$ $A(x)$ for each $x \in 2^{\omega}$, proj being the projection onto the first axis.

Mauldin observed that this result yields immediately the following theorem of Purves [P]: if $f: X \rightarrow Y$ is a Borel map of an analytic set $X$ such that $f$ takes Borel sets in $X$ to Borel sets in $Y$, then the set $\left\{y \in Y: f^{-1}(y)\right.$ is uncountable $\}$ is countable.

Let us notice that Theorem 2.1 yields easily the result of Mauldin, thus providing also a simple proof of Purves' theorem (cf. also a remark made by B. V. Rao in Math. Rev. 83c: 54027).

Let $A$ be as in Mauldin's theorem; one can reduce easily the general case to the case when all sections $A(x)$ are uncountable. Let $F: 2^{\omega} \rightarrow \mathbf{H}\left(2^{\omega}\right)$ be the map described in Theorem 2.1. The map $F$ being measurable with respect to the $\sigma$-algebra of sets open modulo first category sets, there exists a Cantor set $C \subset 2^{\omega}$ such that the restriction of $F$ to $C$ is continuous [K, Chapter II, II]. Then the map $g: C \times 2^{\omega} \rightarrow A$ defined by $g(x, y)=(x, F(x)(y))$ is a required homeomorphism.

4.2. Our last remark is related to a problem stated in [CM 1, p. 88]; the referee has kindly informed us that this proposition has been known to R. D. Mauldin since about 1980.

Proposition. Let $C \subset 2^{\omega} \times 2^{\omega}$ be a coanalytic set whose each vertical section $C(x)$ contains topologically a Cantor set. Then there exists a one-to-one map $f$ of $2^{\omega} \times 2^{\omega}$ 
onto $C$ such that:

(i) $f$ and $f^{-1}$ are measurable with respect to the $\sigma$-algebra $\Delta_{2}^{1}$ of the sets which are simultaneously PCA and CPCA, and

(ii) $f(x, \cdot): 2^{\omega} \rightarrow\{x\} \times C(x)$ is a $\mathscr{B} \mathscr{A}\left(2^{\omega}\right)$-isomorphism for each $x \in 2^{\omega}$.

Let us describe briefly the proof, omitting some standard details. Let

$$
C^{*}=\left\{(x, h) \in 2^{\omega} \times \mathbf{H}\left(2^{\omega}\right): h\left(2^{\omega}\right) \subset C(x)\right\} .
$$

The set $C^{*}$ is coanalytic and hence, by the classical Kôndo Uniformization Theorem, there exists a coanalytic set $T \subset C^{*}$ such that each section $T(x)$ is a singleton. Let

$$
p: T \times \mathbf{H}\left(2^{\omega}\right) \rightarrow 2^{\omega} \text { and } q: 2^{\omega} \times \mathbf{H}\left(2^{\omega}\right) \rightarrow \mathbf{H}\left(2^{\omega}\right)
$$

be the projection onto the first axis and onto the second axis, respectively.

Let us put

$$
M=(p \times \text { id })^{-1}(C) \subset 2^{\omega} \times 2^{\omega}
$$

(id: $2^{\omega} \rightarrow 2^{\omega}$ is the identity), and let us consider a map

$$
k: T \times 2^{\omega} \rightarrow M \text { defined by } k(t, y)=(t, q(t)(y)) \text {. }
$$

The map $k$ is a $\mathscr{B} \mathscr{A}\left(T \times 2^{\omega}\right)$-isomorphism of $T \times 2^{\omega}$ onto the set $k\left(T \times 2^{\omega}\right)$ belonging to $\mathscr{B} \mathscr{A}\left(T \times 2^{\omega}\right)$ and, moreover, $k$ takes Souslin sets (i.e. sets obtained from closed sets by the operation $(\mathscr{A})$ [K, Chapter I, VII]) in the space $T \times 2^{\omega}$ to the Souslin sets in $T \times 2^{\omega}$.

Therefore, the Schröder-Bernstein type argument due to Cenzer and Mauldin, described in $\$ 3$, applied to the map $k$ and the coanalytic set $M$ yields a $\mathscr{B} \mathscr{A}\left(T \times 2^{\omega}\right)$-isomorphism $g$ of $T \times 2^{\omega}$ onto $M$, taking each vertical section $\{t\} \times 2^{\omega}$ into itself. Now, if we put (cf. (*))

$$
f=(p \times \text { id }) \circ g \circ(p \times \text { id })^{-1}: 2^{\omega} \times 2^{\omega} \rightarrow C,
$$

we obtain a map satisfying the assertions of Proposition 4.2.

\section{REFERENCES}

[CM] D. Cenzer and R. D. Mauldin, Measurable parametrizations and selections, Trans. Amer. Math. Soc. 245 (1978), 399-408.

[CM 1] _ Inductive definability: measure and category, Adv. in Math. 38 (1980), 55-90.

[D] C. Dellacherie, Un cours sur les ensembles analytiques, Analytic Sets, Academic Press, New York, 1980.

[K] K. Kuratowski, Topology, Vol. 1, PWN, Warsaw, 1966.

[M] D. Mauldin, Bimeasurable functions, Proc. Amer. Math. Soc. 83 (1981), 369-370.

[P] R. Purves, Bimeasurable functions, Fund. Math. 58 (1966), 149-157.

[S] V. V. Srivatsa, Measurable parametrizations of sets in product spaces, Trans. Amer. Math. Soc. 270 (1982), 537-556.

Dipartment of Mathematics, Warsaw University, PKin IX P., 00 - 901 Warszawa, Poland 